\title{
Clonus: the role of central mechanisms
}

\author{
M R DimitRIJEVIC, P W NATHAN,* AND A M SHERWOOD
}

From the Institute for Clinical Neurophysiology, School of Medicine, Ljubljana, Yugoslavia; The National Hospital for Nervous Diseases, Queen Square, London, England; and Department of Clinical Neurophysiology, Texas Institute for Rehabilitation and Research, Baylor College of Medicine, Houston, Texas, USA

SUMMARY The peripheral and central components in sustained clonus were investigated. The excitability of the motoneurons responding to maintained stretch by clonus was examined by tendon taps, trains of vibratory stimuli and by H-reflex afferent volleys. Every burst of clonic discharge of the motoneurons was shown to be followed by a refractory period, which was followed by a shorter excitatory period. It was concluded that the motoneurons responding clonically to a continuous stretch cannot respond until their excitability has been regained after the refractory period. Attempts to change the rate of clonus in various ways failed to do so. Whether motoneurons of clonic muscles tend to respond maximally to other Ia volleys at the rate of clonus was examined by applying repeated taps to the tendon at rates from 1 to $15 \mathrm{~Hz}$. There was a maximal response at the rate of clonus. Inputs other than those induced by stretch cause clonus; examples of cutaneous inputs causing it are given.

The following facts are known about clonus. The usual rates are 5 to $8 \mathrm{~Hz}$; in any muscle in one patient, the rate tends to remain the same. Like other reflexes induced by stretch, clonus occurs with an appropriate degree of increased muscle tone. When a muscle is exceedingly hypertonic, it does not occur. Clonus is usually present in neurological disorders with abnormally increased tendon jerks, although one sees patients with only slightly increased jerks and very easily obtained clonus. Any mechanism or pharmacological substance that suppresses the excessive jerks and muscle hypertonia tends also to suppress clonus. Clonus arises only when there is a lesion involving the great majority of fibres of the lateral corticospinal tract. This statement is based on personal observations of a large series of patients having operations on the spinal cord with histological examination of the central nervous system (to be published).

One important question is whether clonus is due to a repeated activation of muscle stretch receptors or depends also on a spinal organisation

Addresses for reprint requests: Dr $M$ R Dimitrijevic. TIRR. Texas Medical Center, Houston, Texas 77030, USA and Dr PW Nathan, The National Hospital for Nervous Diseases, Queen Square, London WC1N 3BG.

Accepted 24 October 1979

* Member of the external scientific staff, Medical Research Council, Great Britain. that includes the repetitively activated stretch reflex. The evidence presented here favours the view that the underlying control mechanism of sustained clonus is a central governor of rhythmicity, activated and maintained by Ia synchronised volleys.

Two groups of workers studied clonus in man by recording the activity of single spindle afferents in peripheral nerves by means of fine electrodes put into the nerves. ${ }^{12}$ In Szumski et al's work, ${ }^{1}$ the clonus consisted of a few beats of clonus following a tendon tap in a flexor of the wrist. This unsustained clonus could be prolonged by Jendrassik's manoeuvre. They concluded that in clonus the spindles were abnormally sensitive and that the motoneurons particularly important for manifesting clonus were the dynamic fusimotor neurons. On the other hand, Hagbarth et $a l^{2}$ who studied sustained clonus, found no hyperactivity of the spindles nor were they responding excessively to stretch. They showed that in clonus there was no alpha-gamma co-activation; there were no impulses coming in from the spindles during the contraction phase of the muscle; they occurred only during the muscle's relaxation.

Sustained clonus can occur in neurologically healthy people, as has been shown in the work of Gottlieb and Agarwal. ${ }^{3}$ It can be facilitated by pharmacological substances that increase spindle discharge in stretched muscles. Struppler et $a l^{4}$ obtained it by injecting succinylcholine 
intravenously and Marsden, Meadows and Hodgson $^{5}$ obtained it by injecting adrenalin intraarterially. They also showed that it could be obtained in normal subjects by vibrating the belly and the tendon of the triceps surae "particularly if the evoked tension was great".

The emphasis in recent studies has been on the investigation of the role of the peripheral mechanisms of the stretch reflex. The exception was Walsh ${ }^{6}$ who showed the importance of central components of the reflex in man. The purpose of the present investigation was to elucidate the mechanisms of sustained clonus and to find out the roles of peripheral and central components contributing to the discharge of motoneurons in clonus.

\section{Materials}

This study was carried out on eight healthy subjects and 18 patients. Eleven patients had traumatic division of the spinal cord at various levels, some clinically complete, others partial, and seven had hemiplegia due to vascular lesions in the internal capsule. The paraplegic patients with so-called clinical complete lesions could not move any part of the body below the lesion and there was no sensibility and no thermo-regulatory sweating below the lesion. It should be emphasised that in some of these patients with complete lesions as judged on clinical criteria, physiological investigation has shown evidence of the influence of the brain on segmental reflexes. This has been described elsewhere. ${ }^{7}$

\section{Methods}

The majority of experiments were done on the triceps surae; some were also done on the quadriceps, tibialis anterior, adductors, and biceps femoris and semitendinosus muscles. Clonus was usually evóked in the manner in which it is done in clinical neurology. The foot or patella was pulled up or down and a constant force was maintained. In some experiments the limb was placed with its lateral side downwards on a board, powdered with boric acid to reduce friction between it and the board. At other times it was on its anterior surface over a couch, with the foot protruding beyond the end of the couch.

In one group of experiments, attempts were made to slow the rate of clonus in four ways. One way was to reduce the temperature of the limb by leaving it in ice-water. Another way was to put viscous damping on the movements of the foot by putting it in a stiff jelly, $5 \% \mathrm{w} / \mathrm{v}$ Carbopol
934. The viscosity of this solution was Brookfield $16,0^{\circ} 0$ (cps) $20 \mathrm{rpm}$. The aim was to put drag on the foot and thus slow down the movement. The third way was to add a mass to the foot performing clonus. The fourth way was for one experimenter to induce clonus while the other opposed the movement by pulling the foot in the opposite direction, thus adding force to the antagonists of the movement.

Clonus was recorded by electromyography (EMG), by electrogoniometry, and by a pressure transducer between the foot and a rigid supporting surface. Tendon taps were performed by means of an ADI vibrator, model AV-6; it was applied at various rates up to $14 \mathrm{~Hz}$. It was also used to apply vibration to tendons at rates up to $200 \mathrm{~Hz}$. The head displacement, driven by an clectromagnetic coil, was up to $3 \mathrm{~mm}$, depending on the force on the head.

For nerve stimulation, electrical stimuli, in the form of constant voltage square waves of variable amplitude and pulse-width, were applied through damp, cotton-covered stainless steel electrodes or through Beckman silver-silver chloride electrodes. For H-reflex responses, electrodes were placed over the tibial nerve in the popliteal fossa. A square wave stimulus of $1 \mathrm{~ms}$ duration was used, to favour afferent rather than motor fibres; the stimulus strength was also adjusted to obtain an $\mathrm{H}$-wave and avoid an $\mathrm{M}$-wave. For electric noxious stimuli, $20 \mathrm{~ms}$ trains of $0.2 \mathrm{~ms}$ voltage pulses repeated at $2000 \mathrm{~Hz}$. were applied via Copland-Davies clip-on electrodes.

The movement of the muscle was recorded by several methods. The joint angle was measured by an electrogoniometer. Another method of recording the movement of the foot utilised an air bladder connected to a Statham pressure transducer; this was connected to a signal conditioning device so that this pressure could be recorded with other mechanical and electrical events. The air bladder was between the examiner's hand and the plantar surface of the patient's foot. Recording electrodes were silversilver chloride surface electrodes $1 \mathrm{~cm}$ diameter, $4 \mathrm{~cm}$ apart.

For computation of the average EMG amplitude, a voltage-to-frequency converter technique was used to digitalise the EMG signal after full-wave rectification. Digital integration for an appropriate response interval (usually $20 \mathrm{~ms}$ ) was followed by averaging more than 100 responses. The resultant data points thus computed for each set were normalised to a maximum set value of 100 .

In one series of experiments, the EMG was 
displayed on an oscilloscope by triggering the sweep by the onset of the clonus burst. Electrical stimuli could then be initiated after an adjustable delay. Due to the variability of the shape and amplitude of the EMG discharge, there was a jitter of 5 to $10 \mathrm{~ms}$ in this delay. However, this was less than $10 \%$ of the total interval between two discharges.

\section{Results}

Phases of central excitability during clonus

The first question asked was whether the silent period between two beats of clonus was due to unloading the spindles or to a central refractory period. In these experiments clonus was maintained by continual stretch of the triceps surae while the excitability of the motoneurons performing clonus was examined by putting in three kinds of test stimuli with different delays after the preceding clonic burst. These added stimuli were afferent volleys elicited by tendon taps, H-reflex stimulation, and vibration of the tendon at $80 \mathrm{~Hz}$ with $3 \mathrm{~mm}$ displacement. The experiments were done on a patient with hemiplegia following a vascular lesion of the internal capsule and on 3 patients with lesions of the cervical cord. The sequence of mechanical and electrical events during clonus is shown in fio 1 . It is an excerpt of a continuous recording of sustained clonus maintained by constant stretch on the foot. The times between clonic bursts have been numbered so that they can be referred to. This numbering is mainly based on the recordings in man of Hagbarth, Wallin and Löfstedt $^{8}$ and Hagbarth et al. ${ }^{2}$ Particularly relevant are their recordings of ankle clonus, shown in fig 4 of their second paper.

Point 1 marks the beginning of plantar flexion in response to the contraction of the triceps surae. Between points 1 and 2 , the muscle spindles will be unloaded and their afferents will be silent. At point 2, the limit of plantar flexion is reached. The foot is maintained in plantar flexion between 2 and 3 . After point 3 , the foot dorsiflexes in response to the steady force maintained by the examiner. Between points 1 and 3 , the muscle is contracting. The spindles cease to fire after the next point 1 is reached. In the case illustrated, in which ankle clonus was at a rate of $6 \mathrm{~Hz}$, the duration of muscle relaxation lasted about $65 \mathrm{~ms}$. Among all patients, the range of this period was 50 to $75 \mathrm{~ms}$. In this case, the subsequent EMG burst lasts about 50 $\mathrm{ms}$; the range among all patients was 40 to $70 \mathrm{~ms}$. The silent period in this case was $120 \mathrm{~ms}$; the

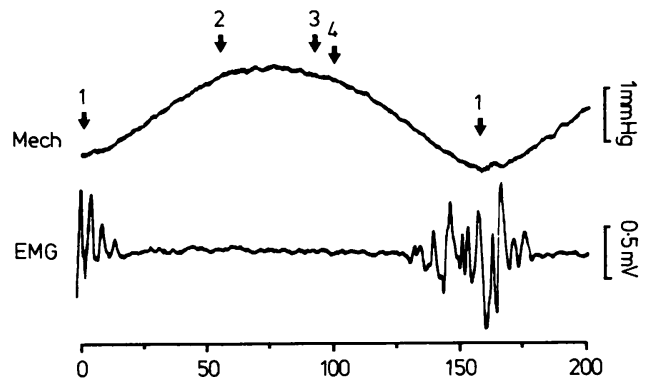

Fig 1 Illustration of two clonic EMG bursts during ongoing clonus. Top channel: mechanogram $=$ foot movement $1 \mathrm{~mm} \mathrm{Hg}$ indicates $1 \mathrm{~mm} \mathrm{Hg}$ pressure in air bladder (see Methods). Bottom channel: EMG in soleus. See text for meaning of numbers. Time in $m s$ in all figures.

range among all patients was 90 to $130 \mathrm{~ms}$.

In the case of the gastrocnemius, it was shown by Dimitrijevic and Nathan ${ }^{9}$ that the latency of jerks was the same in complete cord lesions as in normals. The latency was $30 \pm 3 \mathrm{~ms}$ (shown in their fig 2). The motoneurons will have received the impulses due to this stimulus $15 \mathrm{~ms}$ after the tap, afferent and efferent nerve fibres having about the same conduction velocities. With the H-reflex afferent volley, the latency of the response will be a few ms shorter, as the stimulus is delivered to the nerve in the popliteal fossa. These predominantly Ia afferent stimuli were triggered by the onset of the clonic burst; they were timed to occur at incremental $10 \mathrm{~ms}$ intervals after the onset of the previous EMG burst. They were put into every alternate cycle of clonus, in order to minimise any prolonged effects from the stimulus lasting into the silent period after the next clonic burst.

\section{Testing with tendon tap}

The experiment shown in fig 2 was made in a patient with a partial lesion of the cord at the 6th cervical segment. The top trace shows the Achilles tendon jerk; ankle clonus was not induced. The arrow shows the time of delivery of the tap. A response occurred first when the tap was given $90 \mathrm{~ms}$ after the onset of the previous burst. At $110 \mathrm{~ms}$, the entire EMG burst was replaced by the $T$-wave. Thus this tap fired the entire motoneuron pool and no motoneurons were available for the clonic burst. This response to tapping started a little earlier than the clonic burst would have done. At 130 and $150 \mathrm{~ms}$, large responses were obtained, occurring during the 


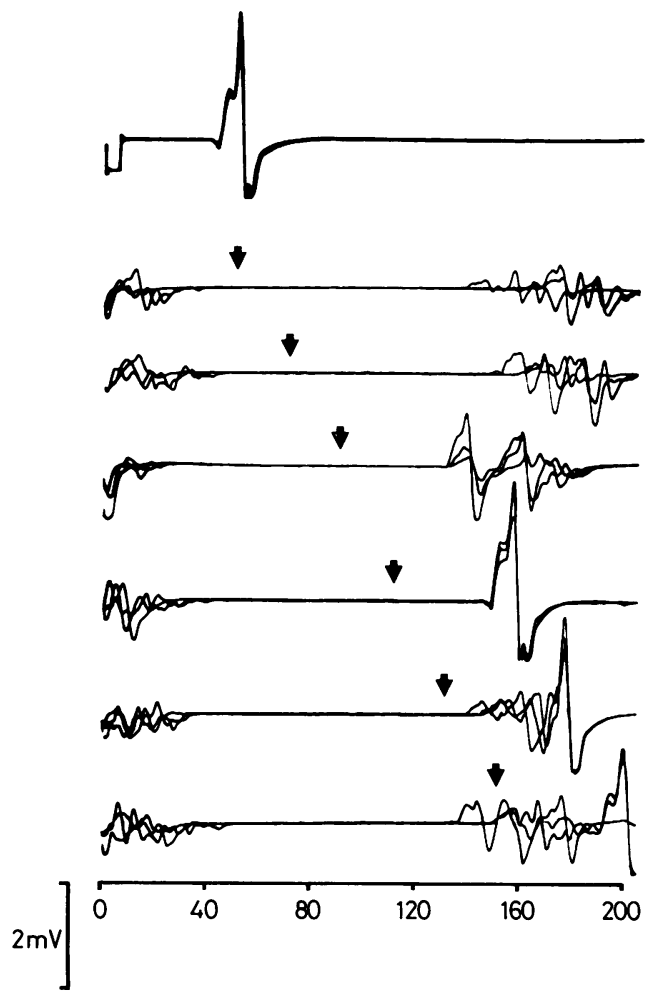

Fig 2 Availability of motor units to a tap on tendo Achillis applied at times shown by arrows. Top trace is the ankle jerk alone. All traces are three superimposed records.

clonic burst. Taps given after $150 \mathrm{~ms}$ elicited no response; nor did they add to the amount nor the duration of the EMG response. This phase of irresponsiveness continued into the early phase of irresponsiveness after the next clonic burst.

Thus the tendon tap produced a response when it was applied while the muscle was not contracting; when it was applied before this, no response was obtained. After the clonic burst, there was a period of $90-100 \mathrm{~ms}$ during which the motoneurons were not activated by interposed Ia afferents. But large responses to tap did occur in the interval from 100 to $150 \mathrm{~ms}$ after the onset of the previous EMG discharge. This was a consistent finding in two spinal cord injury patients and one hemiplegic.

\section{Testing with a train of vibratory stimuli}

In the second group of these experiments, a short tetanic train of vibration was used. The train was too short to induce a tonic vibration reflex and so it did not induce a sustained

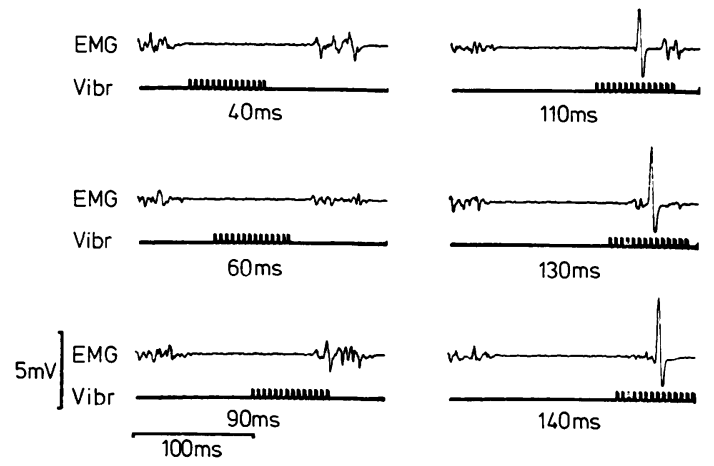

Fig 3 Availability of motor units to vibration on tendo Achillis. The times shown are the times after the onset of the previous clonic EMG burst.

stretch reflex. The reasons for using vibration are that it acts as a continuous conditioning stimulus. The central state could be examined continuously by noting which stimulus of the train elicited a phasic response. The spindle receptors are more sensitive to vibration than to taps on the tendon. Further, vibration excites the spindles irrespective of the state of contraction or relaxation of the muscle.

In a patient with spastic hemiparesis following a vascular lesion in the internal capsule, vibration was applied to the Achilles tendon as a train of 14 impulses lasting $60 \mathrm{~ms}$ at intervals of $10 \mathrm{~ms}$ after the onset of the previous clonic burst. As can be seen from fig 3, no response was obtained until the interval between the beginning of the previous clonic burst and the onset of vibration was $90 \mathrm{~ms}$. A large phasic response occurred when this interval was 110,130 , and $140 \mathrm{~ms}$. If one assumes that the latency to the vibratory stimulus was of the same order as the latency of a jerk-around $30 \mathrm{~ms}$ - the response to the vibration followed the 7 th stimulus at a $90 \mathrm{~ms}$ interval, the 2 nd stimulus at a $110 \mathrm{~ms}$ interval, and the first stimulus at the 130 and $140 \mathrm{~ms}$ intervals. Thus no phasic response to vibration was obtained for 90 to $100 \mathrm{~ms}$ after the onset of the previous clonic burst. The motoneurons could be activated only after 90 $\mathrm{ms}$, and many could be activated at 130 and $140 \mathrm{~ms}$.

Testing with $H$-reflex afferent volleys In the third group of these experiments, an H-reflex afferent volley was used. This electrical stimulus to the tibial nerve also bypasses the input from the spindles by avoiding the contraction and relaxation of the muscle. With 


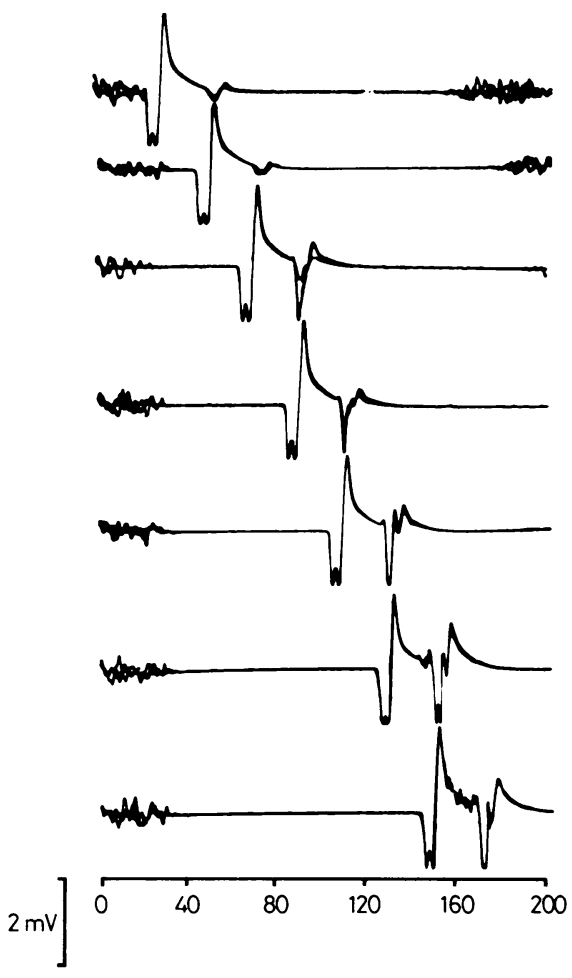

Fig 4 Availability of motor units to $H$-reflex afferent volley. All traces are three superimposed records.

this stimulus, the motoneurons receive a more synchronised volley than with a tendon tap and they receive it a few $\mathrm{ms}$ earlier. The pulse duration and strength of the stimulus was adjusted to obtain a maximal $\mathrm{H}$-wave without an M-wave. On some occasions an M-wave did occur. If it was small, the trace was used, but if it was large, it was discarded. The results of a typical experiment performed on the same patient as was the subject of fig 3 are shown in fig 4. In the first two traces, this stimulus being given 30 and $50 \mathrm{~ms}$ after the onset of the previous EMG burst, the $\mathrm{H}$-wave is seen to be very small. The response was bigger at $90 \mathrm{~ms}$ and reached a peak with a stimulus delay of $60 \mathrm{~ms}$. At $160 \mathrm{~ms}$ it came within the next clonic burst and at $170 \mathrm{~ms}$ at the end of that burst. Neither of these volleys prolonged nor increased the burst. It is concluded that the excitation of Ia afferent fibres cannot activate motor units in this example of clonus for 90 to $100 \mathrm{~ms}$ after the onset of the previous clonic burst nor at 160 to $170 \mathrm{~ms}$ after the burst.
Results of testing with three kinds of Ia afferent volleys

This group of experiments shows that every clonic burst is followed by a refractory period, followed by an excitatory period. The time course varied in accordance with the rate of clonus. The testing of these periods with $\mathrm{H}$ reflex afferents shows that the refractory period is not due to withdrawal of afferent impulses from the spindles.

The curves obtained show that when the motoneurons supplying the triceps surae muscle are discharging clonically, they are refractory to Ia afferent stimuli immediately after the clonic burst and remain so for about $100 \mathrm{~ms}$; they then become excitable for about $60 \mathrm{~ms}$.

It is evident that these centrally organised cyclical changes of excitability are the basis of clonus and that they determine its rate. In clonus, the duration of the periods will not be quite as constant as the periods following synchronised inputs, such as those of tendon taps or H-reflex afferents. For the stretch of the muscle is more gradual and so the response of motor units is less synchronised. The duration of the clonic burst was usually around $60 \mathrm{~ms}$ for the triceps surae and that of the tendon tap $15 \mathrm{~ms}$. It can be inferred that the last activation of muscle spindles before they are unloaded occurs approximately $30 \mathrm{~ms}$ prior to the onset of the last motor unit potential; for there is a prolonged EMG discharge in response to stretch-responding afferents.

\section{Attempts to change the rate of clonus}

One of the features of clonus is the constancy of its rate in any muscle. In five of the patients, sustained ankle clonus was recorded at various times over a period of two years. In any individual patient, the clonus rate of a muscle did not vary by more than $\pm 15 \%$ of its average rate; this was less than $0.7 \mathrm{~Hz}$ deviation from the average rate of $5.3 \mathrm{~Hz}$ in the most extreme case. These slight changes in rate occurred randomly in time; there was no tendency for the rate of decrease or increase over a period of months or years.

The question arises whether the rate of clonus can be changed to values outside this narrow band since, as was shown above, a tap applied with the appropriate delay during clonus could induce the motor units to fire earlier than they would otherwise have done. By shortening the silent period following each EMG discharge. and by increasing the synchronicity of the discharge, the rate of clonus could perhaps be 
increased. Experiments were done in which the afferent volley from the tap on the tendon arrived at the motoneurons at the beginning of the time when they were discharging in the clonic burst. None of these trials changed the rate of clonus by more than the spontaneous variability in this rate, mentioned above.

As the rate of clonus could be changed only to this limited degree by the addition of Ia afferent volleys, experiments were performed to try to change the rate in other ways that acted via peripheral factors. The four ways, described in Methods, of placing the limb in ice-water, of adding a mass to the foot, of moving the foot through a viscous solution, and of the experimenter opposing the movement of the foot carrying out clonus, sometimes changed the amplitude of clonus. But the clonus did not continue at a slower rate; it ceased to occur. Just as it stopped, for two or three beats the rate of clonus was slowed by a maximum of $1 \mathrm{~Hz}$. It appears, then that the constancy and immutability of the rate of clonus in any muscle depend on central organisation.

\section{Tendon reflexes repeated at various rates in clonic muscles}

The question was asked whether in clonically responding muscles a maximal number of motoneurons respond to tendon taps delivered at the rate of clonus. The response of a muscle to a repetitive tapping on its tendon depends on the peripheral factor of the state of the spindle receptors during contraction and relaxation of the muscle and on the central factor of cyclic

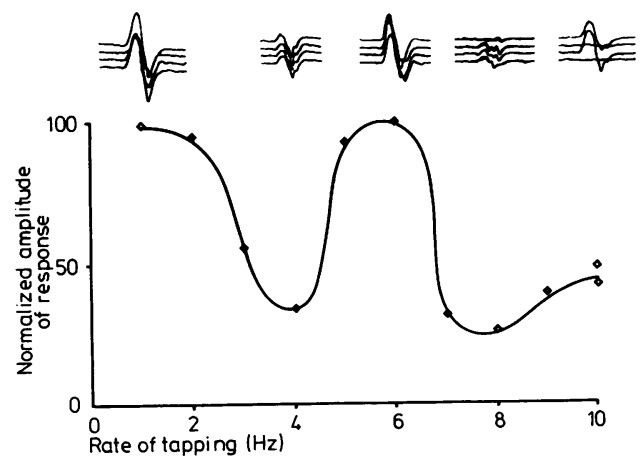

Fig 5 The curve is the averaged amplitude of 100 responses, normalised to the peak value at a tapping rate of $1 \mathrm{~Hz}$, shown at each rate of tapping on tendo Achillis. Representative successive sets of four superimposed traces are given above the curve, at tapping rates of $1,4,6,7$ and $10 \mathrm{~Hz}$. excitability at the time when the volley from the tap is received. To produce repetitive tendon jerks, it was necessary to decrease the tension applied continuously to the muscle to below the threshold for inducing sustained clonus. In fact, the weight of the foot provided adequate tension for obtaining jerks without inducing clonus. Taps were delivered to the tendo Achillis at various rates in three patients with spinal cord lesions, two patients with hemiplegia due to vascular lesions of the internal capsule and three normal subjects. The response to 100 consecutive taps was integrated and averaged, starting $30 \mathrm{~ms}$ after the tap and continuing for $20 \mathrm{~ms}$; the first few responses of every series were omitted, as the response pattern was not immediately stabilised. The data obtained in a patient with an almost complete lesion at the 5th thoracic segment are shown in fig 5. The amplitude of the EMG response, normalised to the amplitude at $1 \mathrm{~Hz}$ and named 100 is shown at each tapping rate. Four sequential sample responses from which the curve was obtained are shown above it. Maximal responses occurred at two repetition rates: $1-2 \mathrm{~Hz}$ and $5-6 \mathrm{~Hz}$. The rate of clonus was $5.6 \mathrm{~Hz}$ in this patient.

This result was typical of all patients: the maximal EMG response occurred when the rate of tapping was at the rate of clonus. A maximal number of motoneurons responded to taps given at a rate of $1 \mathrm{~Hz}$; taps not shown in the figure given at slower repetition rates also produced a maximal number of responding units. With taps given at $1 \mathrm{~Hz}$ or less, each tap is treated as an isolated event; most central activity from the previous tap will have subsided before the next tap is given. All motoneurons did not respond to the taps given at rates of $3,4,7,8,9$ and $10 \mathrm{~Hz}$. In all cases a maximal number of motor units responded to tapping at rates of 5 and $6 \mathrm{~Hz}$, rates similar to the rate of clonus of that muscle in that subject. This suggests that the rate of clonus is a manifestation of a periodicity of excitation affecting motoneurons responding to stretch.

With tapping rates above that of clonus, two kinds of response were seen: a poorly synchronised low amplitude response to each stimulus; or a large synchronised response to alternate taps. There is a $40-50 \%$ response at a tapping rate of $10 \mathrm{~Hz}$, which is twice the rate of tapping of the $95-1 \mathrm{c0} \%$ response at $5 \mathrm{~Hz}$ That peripheral factors were not playing a role is seen by examination of the maximum responsiveness possible from a single motor unit. Fig 6 shows single motor unit responses to tapping rates 


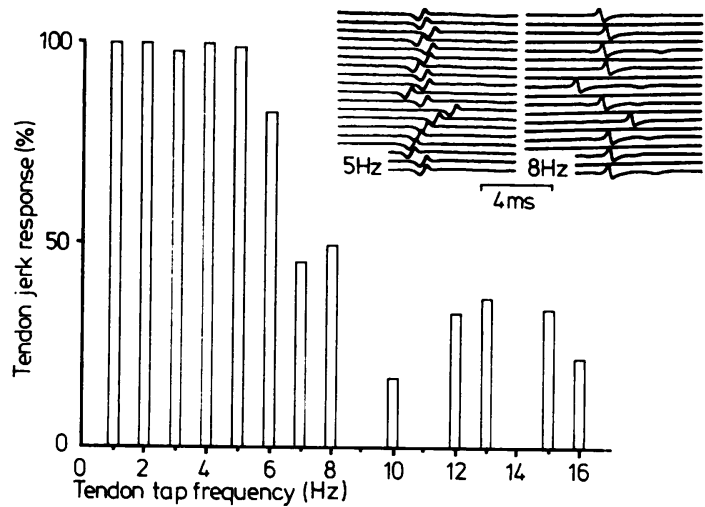

Fig 6 Response of single motor unit of triceps surae to taps repetitively applied at rates of $1-16 \mathrm{~Hz}$. Rates shown in abscissa, percentage of responses to 100 taps in ordinate. Inset in right upper corner; samples of response of single muscle fibres to tapping at 5 and at $8 \mathrm{~Hz}$.

of 1 to $16 \mathrm{~Hz}$. Samples of jittering response are shown at a tapping rate of $5 \mathrm{~Hz}$ and of a response to alternate taps at $8 \mathrm{~Hz}$. While it was possible to excite a single unit by tapping at rates up to the clonus rate, it was impossible to elicit more than a $50 \%$ response beyond that rate, independent of the amount of contraction of the muscle. This can be understood in terms of the centrally induced refractory period. The failure of the whole motor unit population to respond at lower rates (fig 5) was due to induced afterdischarges, as described below.

The response of motor units in spastic patients was examined in more detail. In these patients, a single tap often produced a first response, followed by two bursts of discharge of the motor units. A typical experiment was carried out on a patient with hemiplegia. The foot was plantarflexed sufficiently to prevent sustained ankle clonus. The effect of tapping at different rates on the tendo Achillis is shown in fig 7. The EMG is shown on the top line and the movement of the muscle by the mechanogram trace on the bottom line. The rate of clonus in this muscle was $5.5 \mathrm{~Hz}$. The first few responses at each tapping rate were not recorded; each record shown is a single example taken from a series of taps. The rate of tapping was 2 in a, 3.3 in b, 6 in c, 8 in d, 10 in e and 12.5 in f. Fig 7A shows a first large synchronised EMG response, followed by a second poorly synchronised response of variable amplitude, and a third response of lower amplitude with less synchronisation. Each of these responses is a

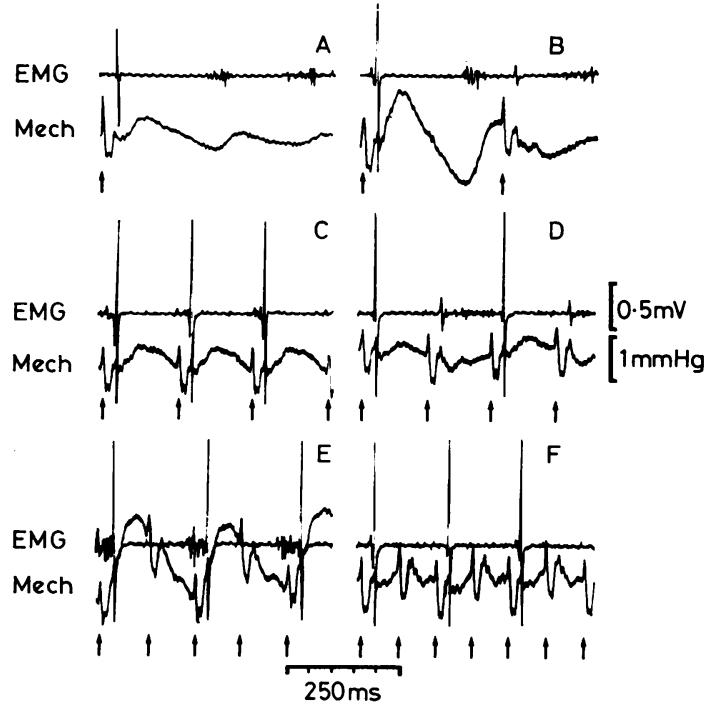

Fig 7 Response of the triceps surae to tap on tendo Achillis at various rates (see text) in a patient who responded to a tap by 3 EMG bursts. Arrows show time of taps. Mechanogram records muscle movement. $1 \mathrm{~mm} \mathrm{Hg}$ means $1 \mathrm{~mm} \mathrm{Hg}$ pressure in air bladder.

muscular contraction, as is shown by the mechanogram. The early response follows the tendon tap with the expected delay of $30 \mathrm{~ms}$; the later responses follow the tap with the same latencies as that of successive beats of clonus. The proposed interpretation of these events is that the second response is a poorly synchronised phasic stretch reflex induced by the stretch when the muscle relaxes at the end of the first response, and the third response is the same, being induced by relaxation following the second response. These responses are occurring at the rate of clonus, $5.5 \mathrm{~Hz}$. These responses are smaller than the first response, being less synchronised. They are less synchronised as the stimulus invoking them is less synchronised than that of a tap.

The largest response occurs when the tap is delivened $30 \mathrm{~ms}$ before or at the same time as the 2nd or 3rd responses to a previous tap; this is seen in fig 7C and E. With a tapping rate of $3 \cdot 3$ $\mathrm{Hz}$ (fig 7B), one tap occurred in the period between two delayed responses and the next tap occurred after the second delayed response. The result was that every alternate tap produced a large response and the other alternate taps produced a small first response with a minimal 
movement and very small delayed responses. It is to be noted that when the 2 nd and 4th and subs?quent alternate taps were delivered, the muscle was relaxing and the spindles were available for stretching. It is apparent that the delayed responses were also inducing refractory periods, similar to those following the first response.

It is clear that the amplitude of the response to tapping depends on the time at which the tap is delivered in relation to the periods of refractoriness and excitability occurring at the time. There is no response to a tendon tap given within 100 to $120 \mathrm{~ms}$ of a previous discharge, whether that discharge is the 1st, 2nd or 3rd response to a tap. When the volley from the tap reaches the motoneurons between 120 and 160 ms after any of these previous discharges, it causes a synchronised response. Inputs received during the refractory period following even poorly synchronised discharges of motoneurons can have no effect, whereas inputs received during the excitatory periods facilitate the discharge that is about to occur. When there is poor synchronisation of the discharge of motor units in the 2nd and 3rd responses to a tap, the new input finds a longer excitatory period during which the response can be facilitated. The input from tapping does not alter the periodicity and re-set the cycle. Tapping cannot activate the system to respond at rates other than the clonic rate.

Why the whole population of motoneurons failed to respond at tapping rates of 3 and $4 \mathrm{~Hz}$ in the experiment illustrated in fig 5 , could be accounted for also on central factors.

These experiments with activating spindle

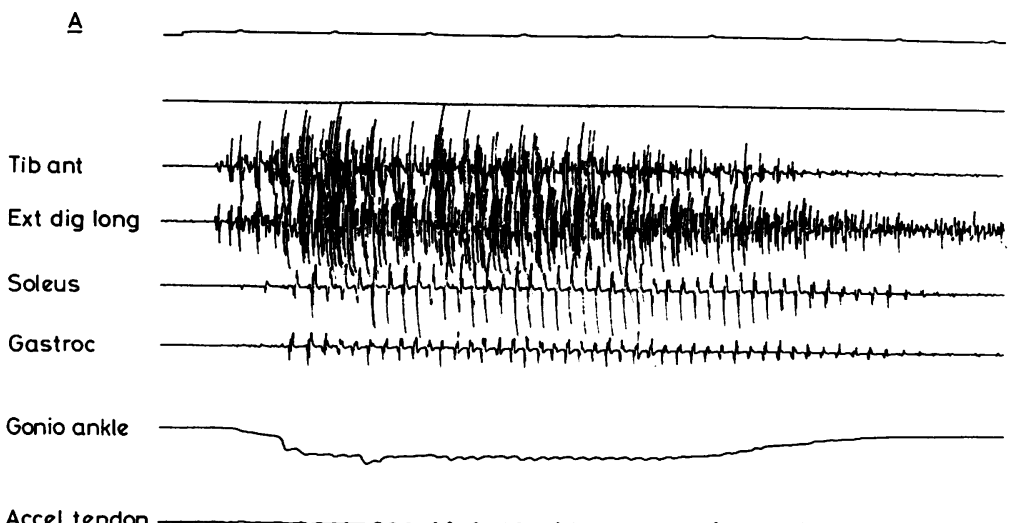

$\underline{B}$

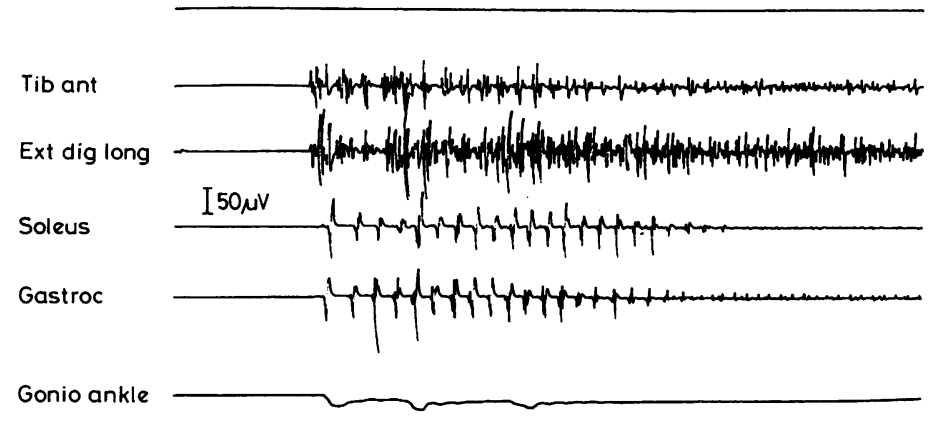

Fig 8 (a) Spray to dorsum of hemiplegic foot. (b) Spray to unaffected foot. Time in top channel, $1 \mathrm{~s}$; this channel is raised during spraying.

Penultimate channel indicates movement of foot, downward trace showing dorsiflexion of foot. Last channel shows movement of tendo Achillis. 
afferents at various repetition rates show that the response to such an input is dependent on the time when the input arrives in relation to the refractory and excitatory periods. The response of the total muscle also depends on the proportion of the motoneurons that is available.

\section{Cutaneous stimuli causing clonus}

Muscles liable to show clonus discharge clonically not only with Ia afferent volleys but also with any exteroceptive stimulus of sudden onset and sufficient intensity. Although clonus is usually and easily induced by stretch of the muscle, it is also induced by other stimuli. The cutaneous stimulation most likely to induce and maintain clonus are noxious stimuli and sudden cold. Merely fixing the clip-on electrodes onto the skin often induced clonus; in some of our patients with spastic paraparesis, this stimulus was so effective in maintaining clonus that it was impossible to use these electrodes, clonus continuing as long as the points were in the epidermis.

An example of clonus being induced by a cutaneous cold stimulus is illustrated in fig 8 . The patients had a right hemiplegia due to a vascular lesion in the internal capsule. Figure 8 shows clonus induced by spraying the dorsum of the right foot with ethyl chloride, and $8 \mathrm{~b}$ that induced by spraying the dorsum of the left unaffected foot. The duration of spraying is shown by the elevation of the time-maker. This cutaneous stimulus to either foot caused clonus in a flexor response. The flexion came on $0.5 \mathrm{~s}$ after spraying the ipsilateral foot and $1.0 \mathrm{~s}$ after spraying the contralateral unaffected foot. These examples show that stimuli that induce a polysynaptic flexor or extensor reflex tend to induce clonus. On some occasions the clonus occurred without any movement of the limb preceding the clonus. A variety of stimuli can elicit clonus when the motoneurons are abnormally excitable. In very spastic patients, a continuous noxious cutaneous stimulus can cause sustained clonus if the muscle is under some tension. Experiments were done in which clonus was maintained as long as a crocodile clip was attached to the foot; it caused clonus of the triceps surae and often of the tibialis anterior as well, and tonic contraction of the other muscles of the leg.

In instances where clonus was induced and maintained by tension on the muscle, the amplitude of the beats would sometimes decline and fade out. Then, cutaneous volleys induced by scratching the skin over the muscle would often provide sufficient input to the spinal cord to restore the amplitude of the clonus.

\section{Discussion}

Whether clonus is entirely due to the peripheral repetitive activation of the spindle stretch receptors or depends also on a spinal governor mechanism created by the cyclic activation of the stretch reflex is the main question we have investigated.

When single taps are given to the Achilles tendon of spastic patients, the duration of the reflex contraction and relaxation of the muscles is around $300 \mathrm{~ms}$. If clonus were simply a repetitive stretch reflex with no regulation of rate, it could occur at a rate of 3 cycles a second, given time for a complete cycle of contraction and relaxation of the triceps surae; or it might occur at any rate up to $14 \mathrm{~Hz}$, when tetanus occurs. In fact, clonus, as is well known, occurs within a narrow band of frequencies from 5 to $8 \mathrm{~Hz}$. This fact alone shows that central regulatory factors are operating.

If the rhythm of clonus is determined by the availability of the spindle receptors during the phases of contraction and relaxation of the muscle, it should be possible to change the rate of clonus by exciting these receptors at different rates. And if clonus is due to the modulation of a continuous stretch on the muscle by the contraction and relaxation of the muscle itself, then the rate of clonus would be affected by the mass of the part being moved, by muscular viscosity, and by the force applied to the part. We were unable to change the rate of clonus; nor was this achieved by Walsh. ${ }^{6}$ nor by Sherwood and Dimitrijevic $^{10}$ using other methods. We also did not change the rate of clonus by altering the force applied to the part.

The investigations reported here show that between two bursts of clonus there is a refractory state within the spinal segments followed by an excitatory state. The motoneurons cannot respond to continuing tension put on the muscle until they have become adequately excitable following the refractory period. This periodicity can be modified only during the very brief time when the refractory period is changing to the excitatory period; Ia inputs can then influence the period.

As the duration of the EMG burst in sustained clonus depends on the duration of spindle activity, the question arises whether this duration can be prolonged by extending the period of activation of the spindles. We have found that it cannot be, since delivering additional stimuli in the form of tendon taps, vibration or electrical depolarisation of the Ia fibres during the period immediately following the excitable phase does 
not result in any activation of motor units involved in the clonus activity. This demonstrates the complete unresponsiveness of these pathways during this time.

The sudden decline in excitability after the end of the clonus EMG burst and the long duration of the refractory period, together with the rapid recovery of excitability, suggest the presence of an active inhibitory process. Brune and Schenck ${ }^{11}$ examined the silent period between two clonic bursts by putting in random H-reflex volleys. Some of their $\mathrm{H}$-waves were true $\mathrm{H}$-waves and others had an intensity to cause large $\mathbf{M}$-waves. They also found a loss of responsiveness to stimulation between the EMG bursts. They concluded that the cessation of motoneuron activity at the beginning of the silent period was due to refractoriness of the motoneurons after firing, combined with Renshaw inhibition, and that the rest of the period was due to withdrawal of excitation coming from the spindle afferents. Struppler, Burg and Erbel ${ }^{12}$ have concluded that these factors include not only muscle spindle unloading, but also autogenetic inhibition by Golgi afferents, recurrent inhibition by Renshaw cells, and the refractory phase of the motoneurons.

An experiment demonstrating the importance of spinal mechanisms in clonus was performed by Walsh. ${ }^{13}$ To a foot performing clonus, he added a periodic force. Modulation of this applied force was at frequencies of 5 and $5.5 \mathrm{~Hz}$ while the clonus rate was $6 \mathrm{~Hz}$. Beat frequencies corresponding to the difference in rate of clonus and the force modulation frequency $(0.5$ and $1 \mathrm{~Hz})$ occurred. He concluded ${ }^{613}$ from this and from other evidence that the rhythm of clonus is "centrally tuned".

Other investigators who favoured the central excitatory state as being the essential factor in clonus are Wachholder and Altenburger ${ }^{14}$ and Brune and Schenck. ${ }^{11}$ Wachholder and Altenburger ${ }^{14}$ showed that the latency of the first beat of the clonus was the same as that of the stretch reflex; they claimed that this time relation was not maintained during sustained clonus. They therefore concluded that clonus is started by stretch and that the rhythmical discharge is maintained by central factors. We found that the latency of the EMG response was correct throughout sustained clonus for each burst to be a response to Ia afferent volleys.

The features of clonus that have led us to put most weight on central factors were the findings that a maximal number of motoneurons respond to repetilive tendon taps at the rate of clonus of that muscle, and that the mechanical activity of the contraction of the muscle adjusted itself to that rate. Furthermore, the rate of clonus is relatively constant, and the direction of the small shifts in rate is peripherally controlled. We have shown that an important mechanism in this rate regulation is the periodic repetition of excitatory and refractory states corresponding to the cyclic activation of clonus.

That clonus depends essentially on factors in the periphery and minimally on central factors is the view of investigators to be discussed now. Bernhard and Therman ${ }^{15}$ studied the triceps surae of the cat in a decerebate state. Decerebation causes a particular kind of hyperexcitability of stretch reflexes. Using passive movements of the limbs and muscles, they showed that the continuous muscle contraction could be changed into a rhythmical contraction. When a tonically contracting extensor muscle was passively flexed, the contraction became rhythmically intermittent: there were bursts of EMG actively separated by silent periods. When the new position of the limb was taken up, the tonic activity recurred. It was the changing proprioceptive input from the muscle itself, from its synergists or its antagonists, that changed the tonic activity into bursts of discharges separated by silent periods. Single motor units were then discharging within limited frequency bands. They repeated the observations of Denny-Brown ${ }^{16}$ that the application of passive tension of the muscle will start rhythmical activity of a single unit in the extensor muscle of a decerebrated cat. This work then shows that in the decerebrate cat proprioceptive input from the limb can start rhythmical discharges of motor units while the limb is being moved.

A characteristic of clonus is the synchrony of the motor discharge. It may not be marked for the first three or four beats of clonus, then it becomes established. The synchrony of discharge occurs although peripheral factors contributing to clonus, such as the input from the spindles, the geometry of the muscle and the rate of relaxation of the parts of the muscle, are far from being synchronised. This shows that the reflex is rigidly controlled in time and also in spatial extent in the motor unit pool. The discrepancy between the scattered peripheral factors and the synchronised motor unit response indicates that central mechanisms are playing a major role.

It appears to be that when an adequate volley of impulses reaches the motoneurons liable to respond clonically, it fires them synchronously; and it is the synchrony of firing that starts clonus. This synchronous response occurring in the same 
rhythm determines the rate of clonus. The synchronous firing of these motoneurons is also started by a tendon tap, and so one tendon tap can initiate several bursts of clonus.

The question remains unanswered why certain motoneurons tend to fire synchronously rather than asynchronously in response to a continuous input. Whatever the cause, there is no absolute difference in the organisation of these motoneurons, for they often respond tonically; and motoneurons of other muscles that habitually respond tonically can be entrained to respond clonically when the whole limb is being rhy.hmically moved in a clonic rhythm.

We conclude that the hypothesis that clonus is essentially a peripheral phenomenon, being a repeated stretch reflex, is inadequate. The evidence favours the hypothesis that it relies on a central, spinal generator or controller. This generator should be conceived as a transitory functional organisation, made up of the following elements: the movement of the muscle and parts of the limb, the proprioceptive volleys from the limb, the segmental reflex activity which is influenced by peripheral, propriospinal and suprasegmental mechanisms, when these are present. The features of this transitory functional organisation include:

(a) cyclic, regular activation at a fixed phase by Ia afferent volleys;

(b) activation of motoneurons and coactivation of convergent interneurons;

(c) functional isolation of segmentally organised repetitive stretch reflex activity from the disruptive influence of extraneous volleys;

(d) maintenance of the motoneuron pool excitability;

(e) the resultant excitatory-ref ractory cycles which sustain and regulate the oscillatory movements.

These features are consistent with the idea of a spinal governor, rather than a peripheral mechanical controller. While the peripheral input is essential for the reactivation of the cyclic bursts, the spinal mechanism tightly controls the overall activity. The periods of refractoriness that are the cause of the intermittent discharge of clonus must be due to inhibition of the motoneurons and/or interneurons. The period of refractoriness is too long to be due to Renshaw inhibition; and Veale, Rees and Mark $^{17}$ have shown that this early recurrent inhibition is absent in spasticity in man. What does cause this inhibition of certain motoneurons and related interneurons remains unknown. We have not presented any evidence on this matter, as in this paper we are concerned only with the relative importance of peripheral and central mechanisms in the genesis of clonus.

As clonus is due to mechanisms within the local segments of the spinal cord in spasticity, it might be expected to see some indications of its presence in normal subjects. This is the case, as we mentioned in the introduction; and it is common experience that when one sits and puts the correct amount of tension on the leg, a clonic movement of the leg can easily be induced and maintained. Clonus in normal subjects has been studied by Gottlieb and Agarwal. ${ }^{3}$. This clonus had the same characteristics as clonus in patients, showed the same relatively narrow band of frequencies based on the same timing of EMG bursts, and the same relative independence of loading on the limb. To obtain clonus in normal subjects, apart from the methods mentioned before of injecting substances that increase spindle discharge, increased muscle tension has to be maintained, and other inputs to the motoneurons such as those necessary for voluntary movements have to be prevented. Gottlieb and Agarwal induced flexion and extension of the foot at the ankle joint by a foot-plate connected to a motor, which oscillated the foot at repetition rates between 5 and $8 \mathrm{~Hz}$. With these rates of driven oscillation, there were "large excursions of the foot with very small applied torques and strong synchronous activation of the soleus muscle and, sometimes, of the anterior tibial muscle". And what was more interesting, they often found that when the motor was turned off, clonus continued for more than a minute without any tension applied to the foot; it could have continued longer but the subjects then stopped it.

When there are lesions dividing corticospinal tract fibres, these segmental circuits, present in the normal, become more independent and less influenced by volleys of impulses from the brain. In the absence of supraspinal inputs, the firing threshold of motoneurons becomes low. When clonically reacting motoneurons are facilitated by Ia affergent inputs, they will respond clonically to any input. We often found, as have so many other investigators, that clonus could be induced by the non-specific descending facilitation caused by Jendrassik's manoeuvre. Further, clonus could be induced by inputs in flexor reflex afferents. It is also true that stimuli activating these pathways can stop clonus. Which event occurs depends, among other factors, on the position of the stimulus invoking flexion in relation to the muscle responding clonically.

In conclusion, it is now clear why the question of the relative importance of the central and the 
peripheral mechanisms in defining clonus has been difficult to answer. As we have shown, the rigidly determined cyclic excitatory-inhibitory phases of the segmental organisation determine the rate of clonus. Yet excitation for the maintenance of these oscillations must be provided by properly timed muscle spindle afferent volley. The interaction of the muscle contraction-relaxation cycle with the excitatoryinhibitory cycle of the repetitive stretch reflex automatically adjusts the reflex contraction duration to the value determined by the segmental governor.

We thank our colleagues from the departments of neurology, neurosurgery and orthopaedic surgery at the University Hospital of Ljubljana, of the National Hospital for Nervous Diseases, Queen Square, London, and of the Texas Institute for Rehabilitation and Research, Houston, for referring their patients to us for our study. This work was made possible through the financial support of the Boris Kidric Fund of Slovenia, the Medical Research Council of Great Britain, a grant from the Bob and Vivian Smith Foundation of Houston, and Rehabilitation Service Administration Grants 13-P-59275/6 and 16-P-56813/6.

\section{References}

1 Szumski AJ, Burg D, Struppler A, Velho F Activity of muscle spindles during muscle twitch and clonus in normal and spastic human subjects. Electroencephalogr Clin Neurophysiol 1974; 7:589-97.

2. Hagbarth KE, Wallin G, Löfstedt L, Aquilonius SM. Muscle spindle activity in alternating tremor of Parkinsonism and in clonus. J Neurol Neurosurg Psychiatry 1975; 38:636-41.

3 Gottlieb GL, Agarwal GC. Physiological clonus in man. Exp Neurol 1977; 54:616-21.

4 Struppler A, Schulte FJ, Scheininger R, Kukku
M. Eine elektromyographische Untersuchung bei Spastik und Rigor. Deutsch Z Nervenheilk 1961; 183:134-47.

5 Marsden CD, Meadows JD, Hodgson HJ. Observations on the reflex response to muscle vibration and its voluntary control. Brain 1969; 92:829-46.

6 Walsh EG. Clonus: beats provoked by the application of a rhythmic force. J Neurol Neurosurg Psychiatry 1976; 39:266-74.

7 Dimitrijevic MR, Spencer WA, Trontelj JV, Dimitrijevic M. Reflex effects of vibration in patients with spinal cord lesions. Neurology 1977; 27:1078-86.

8 Hagbarth KE, Wallin G, Löfstedt L. Muscle spindle activity in man during voluntary fast alternating movement. J Neurol Neurosurg Psychiatry 1975; 38:625-35.

9 Dimitrijevic MR, Nathan PW. Studies of spasticity in man: 2, Analysis of stretch reflexes in spasticity. Brain 1967; 90:333-58.

10 Sherwood AM, Dimitrijevic MR. Clonus: a simplistic model of a complex system. In: Proc $29 \mathrm{~A}$ Conf Eng Med Biol 1976; 18:278.

11 Brune HF, Schenck E. Neurophysiologische Untersuchungen über den Klonus bei Spastikern. Arch Psychiatr Nervenkr 1960; 201:65-80.

12 Struppler A, Burg D, Erbel F. The unloading reflex under normal and pathological conditions in man. In: Desmedt JE, ed. New Developments in Electromyography and Clinical Neurophysiology, vol 3. Basel: Karger, 1973; 603-17.

13 Walsh EG. Ankle clonus-an autonomous central pacemaker? J Physiol 1971; 212:38-9.

14 Wachholder K, Altenburger H. Experimentelle Untersuchungen zur Entstehung des Fussklonus. Deutsch $Z$ Nervenheilk 1925; 84:117-21.

15 Bernhard CG, Therman PO. Rhythmical activity of motor units in myotatic reflexes. Acta Physiol Scand (suppl) 1947; 14:1-14.

16 Denny-Brown $D$. On the nature of postural reflexes. Proc $R$ Soc [Biol] 1929; 104:252-301.

17 Veale JL, Rees S, Mark RF. Renshaw cell activity in normal and spastic man. In: Desmedt JE, ed. New Developments in Electromyography and Clinical Neurophysiology, vol 3. Basel: Karger, 1973; 523-37. 